\title{
RESONANCE TONGUES AND SPECTRAL GAPS IN QUASI-PERIODIC SCHRÖDINGER OPERATORS WITH ONE OR MORE FREQUENCIES. A NUMERICAL EXPLORATION
}

\author{
JOAQUIM PUIG AND CARLES SIMÓ
}

\begin{abstract}
In this paper we investigate numerically the spectrum of some representative examples of discrete one-dimensional Schrödinger operators with quasi-periodic potential in terms of a perturbative constant $b$ and the spectral parameter $a$. Our examples include the well-known Almost Mathieu model, other trigonometric potentials with a single quasi-periodic frequency and generalisations with two and three frequencies. We computed numerically the rotation number and the Lyapunov exponent to detect open and collapsed gaps, resonance tongues and the measure of the spectrum.

We found that the case with one frequency was significantly different from the case of several frequencies because the latter has all gaps collapsed for a sufficiently large value of the perturbative constant and thus the spectrum is a single spectral band with positive Lyapunov exponent. In contrast, in the cases with one frequency considered, gaps are always dense in the spectrum, although some gaps may collapse either for a single value of the perturbative constant or for a range of values.

In all cases we found that there is a curve in the $(a, b)$-plane which separates the regions where the Lyapunov exponent is zero in the spectrum and where it is positive. Along this curve, which is $b=2$ in the Almost Mathieu case, the measure of the spectrum is zero.
\end{abstract}

To Prof. Russell Johnson in his 60th anniversary, with a deep appreciation for his outstanding works

\section{INTRODUCTION}

In this paper we will explore numerically the structure of the spectrum of some paradigms of Schrödinger operators with quasi-periodic potentials with one or more irrational frequencies. More precisely, we will consider the following type of discrete Schrödinger operators on $l^{2}(\mathbb{Z})$,

$$
\left(H_{b V, \phi} x\right)_{n}=x_{n+1}+x_{n-1}+b V(\omega n+\phi) x_{n}, \quad n \in \mathbb{Z},
$$

where $V:(\mathbb{T})^{d}=(\mathbb{R} / 2 \pi \mathbb{Z})^{d} \rightarrow \mathbb{R}$ is a potential, $d \geq 1, b \in \mathbb{R}$ is a coupling parameter, $\phi \in \mathbb{T}^{d}$ is a phase and $\omega=\left(\omega_{1}, \ldots, \omega_{d}\right) \in \mathbb{R}^{d}$ is an irrational frequency

Date: July 13, 2010. To appear in J. Dynam. Differential Equations.

2000 Mathematics Subject Classification. Primary: 37B55, 35J10.

Key words and phrases. Quasi-Periodic Schrödinger operators, Quasi-Periodic Cocycles and Skew-products, Spectral Gaps, Resonance Tongues, Rotation number, Lyapunov exponent, Numerical explorations.

The research of J.P. has been supported by grant MTM2006-00478. The research of C.S. has been supported by grant MTM2006-05849/Consolider (Spain). The massive computations (a total number of iterates close to $10^{16}$ ) have been carried out at the EIXAM and MAYA clusters of the UPC team on Dynamical Systems and the HIDRA cluster of the UB team on Dynamical Systems. We also want to thank Håkan Eliasson for fruitful discussions. 
vector in the sense that

$$
\langle\mathbf{k}, \omega\rangle=k_{1} \omega_{1}+\ldots+k_{d} \omega_{d} \notin 2 \pi \mathbb{Z} \text { for all } \mathbf{k}=\left(k_{1}, \ldots, k_{d}\right) \in \mathbb{Z}^{d} \backslash\{\mathbf{0}\},
$$

that is, if $\gamma=\omega / 2 \pi \in \mathbb{R}^{d}$ is rationally independent. In all cases we shall assume, without loss of generality, that the potential $V$ has zero average.

These operators have been focus of intensive research over the last decades and much is known about the structure of the spectrum of these operators, being Cantor spectrum (i.e. gaps in the spectrum are dense) the object of much of this research. Note that due to the rational independence of the frequency vector $\omega$, the spectrum of these operators is independent of $\phi$. Many of the recent advances are based on the study of the dynamics of the eigenvalue equation associated to (1.1), namely

$$
x_{n+1}+x_{n-1}+b V(\omega n+\phi) x_{n}=a x_{n}, \quad n \in \mathbb{Z},
$$

where $a$ is a real parameter, usually called the energy.

In the case of a real analytic potential $V$ (in the examples we study, we will further restrict to simple trigonometric polynomials) and fixed Diophantine frequencies (see [Joh91, FJP02, CF08, ABD09] for genericity results in spaces of functions of lower regularity), there are two situations where such Cantor spectrum has been proved and studied (at least generically). The first one is for "small" values of $|b|$, where genericity of Cantor spectrum follows from the genericity of gap opening [MP84, Eli92, BPS03, PS06], a property also shared by periodic continuous systems (i.e., ODE of Hill-Schrödinger type) [MW79, BS00]. Note that, as in this last case, it is possible to produce real analytic potentials where all but a finite number of spectral gaps are closed and, therefore, they do not have Cantor spectrum [DJ87].

Besides, the well-known Almost Mathieu case, where $V(\theta)=\cos \theta$, displays Cantor spectrum for all $b \neq 0$ [CEY90, Pui04, AJ05]. The type of gap opening of this model is significantly different from the previous above, since it appears also for "large" values of $b$, where the spectral behaviour is very different from their periodic ODE analogues: the Lyapunov exponent is positive in the spectrum and there is Anderson localization (see the review in [Jit07]). We recall that in the periodic case the spectrum is the union of finitely many interval bands (or countably many in the periodic ODE case). The second type of Cantor spectrum can also be extended to real analytic and non-constant potentials $V$ with a single Diophantine frequency $(d=1)$ and for all values of $|b|$ larger than a certain constant [Sin87, GS08, HP].

Although the first paradigm of Cantor spectrum holds for multifrequency quasiperiodic and real analytic potentials with small $b$, the structure of gaps for large $b$ is much less clear for these multifrequency models. For two-frequency "nondegenerate" potentials and $|b|$ large enough, work by Chulaevsky and Sinai [CS89] indicates that the spectrum might consist of a single spectral band with no gaps at all. This motivates the following goals of our present numerical study:

(1) To detect the different dynamics in terms of the Lyapunov exponent and the rotation number.

(2) To confirm this picture in some selected models of quasi-periodic Schrödinger operators with one or more frequencies.

(3) To describe the transition from Cantor spectrum, for small values of $b$, to a single spectral band, for large values of $b$ in terms of gap closing in the $d \geq 2$ case.

(4) To compute the measure of the spectrum in the different cases. 
(5) To stress on the differences of the behaviour compared to the case of one frequency.

\section{Methods}

2.1. Dynamical formulation: skew-products, cocycles and reducibility. As mentioned in the Introduction, many of the recent results and techniques for quasi-periodic discrete Schrödinger operators are based on the fruitful interaction between the spectral properties of (1.1) and the dynamical properties of the eigenvalue equations (1.2). It is convenient to write (1.2) as a first order system

$$
\left(\begin{array}{c}
x_{n+1} \\
x_{n}
\end{array}\right)=\left(\begin{array}{cr}
a-b V\left(\theta_{n}\right) & -1 \\
1 & 0
\end{array}\right)\left(\begin{array}{c}
x_{n} \\
x_{n-1}
\end{array}\right), \quad \theta_{n+1}=\theta_{n}+\omega
$$

being $\theta_{n} \in \mathbb{T}^{d}$ new angular variables. Such first-order systems are usually called quasi-periodic skew-products. The dynamical evolution of the vector $v_{n}=\left(x_{n+1}, x_{n}\right)^{T}$ and the angles $\theta_{n}$ can be seen as the iteration of a quasi-periodic cocycle on $\mathbb{R}^{2} \times \mathbb{T}^{d}$, which is the map

$$
(v, \theta) \in \mathbb{R}^{2} \times \mathbb{T}^{d} \mapsto\left(A_{a, b}, \omega\right)(v, \theta)=\left(A_{a, b}(\theta) v, \theta+\omega\right) \in \mathbb{R}^{2} \times \mathbb{T}^{d},
$$

where

$$
A_{a, b}(\theta)=\left(\begin{array}{cr}
a-b V(\theta) & -1 \\
1 & 0
\end{array}\right) .
$$

When the frequency vector $\omega$ is rational, the discrete skew-product is periodic and, by composition, it can be reduced to a new one with constant matrix (but it depends on the initial phase $\theta_{0}$ ). In the ODE case this can be achieved by Floquet theory and the system can be reduced to constant coefficients. Although quasi-periodic systems need not to be reducible, one can sometimes "reduce" them to constant coefficients. More precisely, we say that a cocycle $(A, \omega)$ is reducible to constant coefficients whenever there is a continuous transformation $Z: \mathbb{T} \rightarrow$ $S L(2, \mathbb{R})$ such that

$$
A(\theta) Z(\theta)=Z(\theta+\omega) B, \quad \theta \in \mathbb{T}^{d},
$$

where $B \in S L(2, \mathbb{C})$ is a constant matrix, called the Floquet matrix. Whenever a skew-product like (2.1) is reducible to constant coefficients, a fundamental matrix for the system can be expressed in terms of the following Floquet representation

$$
X_{n}\left(\theta_{0}\right)=Z\left(n \omega+\theta_{0}\right) B^{n} Z\left(\theta_{0}\right)^{-1} X_{0}\left(\theta_{0}\right)
$$

for any initial phase $\theta_{0} \in \mathbb{T}^{d}$. Reducibility in quasi-periodic ODE systems which are perturbations of cocycles with constant coefficients has been studied in [BMS76, JS92, Eli92].

The dynamics of reducible systems can be well understood in terms of the eigenvalues of the corresponding Floquet matrices. For instance, if its trace is greater than two in absolute value the skew-product has no bounded solutions in $\mathbb{Z}$ apart from the trivial one. That is, it is uniformly hyperbolic [SS78]. In general to measure the exponential growth of solutions, one can use the (upper) Lyapunov exponent,

$$
\beta(a, b)=\lim _{N \rightarrow \infty} \frac{1}{N} \int_{\mathbb{T}}\left\|A_{a, b}((N-1) \omega+\theta) \cdots A_{a, b}(\omega+\theta) A_{a, b}(\theta)\right\| d \theta,
$$

which exists by Kingman subadditive ergodic theorem [Kin68]. Whenever a system has positive Lyapunov exponent but it is not uniformly hyperbolic, we will call it 
nonuniformly hyperbolic. Note that systems reducible to constant coefficients are either uniformly hyperbolic or have zero Lyapunov exponent.

2.2. Rotation number, spectral gaps and resonance tongues. The rotation number is a useful object for the study of the spectrum of Schrödinger operators with quasi-periodic potential. To define it, take $\left(x_{n}\right)_{n \in \mathbb{Z}}$ a non-trivial solution of (1.2), for some fixed $a, b, \phi$. Let $S(N)$ be the number of changes of sign of such solution for $1 \leq n \leq N$, paying attention to the cases in which some $x_{n}=0$. Then the limit

$$
\rho(a, b)=\lim _{N \rightarrow \infty} \frac{S(N)}{2 N} \in[0,1 / 2]
$$

exists, it does not depend on the chosen solution $x$, nor on $\phi$ and it is called the rotation number [DS83] originally introduced for the continuous case by Johnson and Moser [JM82] (see also the definition of the fibered rotation number for $S L(2, \mathbb{R})$ cocycles by Herman [Her83]).

Remark 2.1. If in (2.1) we consider $b=0$, the constant matrix is conjugated to a rotation of angle $2 \pi \alpha$ if $a=2 \cos (2 \pi \alpha)$, while it is hyperbolic (resp. hyperbolic with reflection) if $a>2$ (resp. $a<-2$ ). It is natural to define its rotation number as $\rho=\alpha$ if $|a| \leq 2, \rho=0$ (resp. $\rho=1 / 2$ ) for $a>2$ (resp. $a<-2$ ). This agrees with the definition (2.4) and we shall use it for the numerical computations, although the rotation number is then decreasing as a function of $a$. One could also define $\operatorname{rot}(a, b)=1-2 \rho(a, b)$ so that rot is a function increasing from 0 to 1 when $b=0$ and $a$ ranges in $\mathbb{R}$. We shall use this function $\operatorname{rot}(\cdot, b)$ in some plots. In other words, there are several possible "natural" definitions of the rotation number.

Remark 2.2. If we look at the argument of vectors in (2.1), we obtain a "nonautonomous" circle map. In contrast with the autonomous case which, for good regularity conditions and Diophantine (or Brjuno) conditions on the rotation number, is conjugated to a rigid rotation, the iterates do not appear in the same order as in a rigid rotation. This prevents the use of methods based on the order of the iterates in $\mathbb{S}^{1}$ (see, e.g., the Appendix in [SNS09]).

For a fixed potential $V: \mathbb{T}^{d} \rightarrow \mathbb{R}$ and irrational frequency vector $\omega \in \mathbb{R}^{d}$, the rotation number is a continuous function of $(a, b) \in \mathbb{R}^{2}$. Also, for a fixed $b$, the spectrum of (1.1), denoted as $\sigma_{b}$, is the set of $a_{0} \in \mathbb{R}$, such that the map $a \mapsto \operatorname{rot}(a, b)$, which never decreases, is not locally constant at $a_{0}$. The values of $\operatorname{rot}(a, b)$ in these sets of constancy are of a very particular form, as the Gap Labelling theorem, by Johnson and Moser [JM82], predicts. Indeed, if $I$ is an open spectral gap, that is non-void interval in the resolvent set of (1.1), then there is a multi-integer $\mathbf{k}=\left(k_{1}, \ldots, k_{d}\right) \in \mathbb{Z}^{d}$ such that the rotation number is resonant

$$
\operatorname{rot}(a, b)-\langle\mathbf{k}, \gamma\rangle \in \mathbb{Z}
$$

for all $a \in I$.

From this theorem, we conclude that the resolvent set is the disjoint union of countably (or finitely) many open intervals called spectral gaps, possibly void, and which can be uniquely labelled by an index $\mathbf{k}$ called the resonance. By abuse of notation we will say that, for a fixed $b$, a value $a$ is a collapsed gap whenever its rotation number is resonant and rot is strictly increasing at that value of $a$, and we will distinguish it from non-collapsed gaps which are the actual open intervals in the resolvent set (whose rotation number is thus resonant). As said in the introduction, 
open gaps and closed gaps can coexist and Cantor spectrum occurs whenever open spectral gaps are dense in the spectrum.

The global structure of spectral gaps for varying $b$ can be better understood in terms of resonance tongues, which are the connected components of constancy of the rotation number in the $(a, b)$-plane. More precisely, given a resonant rotation number, $\operatorname{rot}_{0}$ with $\operatorname{rot}_{0}-\langle\mathbf{k}, \gamma\rangle \in \mathbb{Z}$, the resonance tongue associated to $\operatorname{rot}_{0}$ (or, equivalently, to $\mathbf{k})$ is the set of $(a, b) \in \mathbb{R}^{2}$ for which $\operatorname{rot}(a, b)=\operatorname{rot}_{0}$. Using this notation, spectral gaps are the "slices" of resonance tongues for $b$ constant. The slice for a fixed rot $_{0}$ has some amplitude, which depends on $b$, defined as the length $\ell_{\text {rot }_{0}}(b)$ in $a$ of the interval for the given value of $b$, which can become zero at some values of $b$. Assume $\ell_{\text {rot }_{0}}\left(b_{1}\right)=\ell_{\text {rot }_{0}}\left(b_{2}\right)=0$ for $b_{1}<b_{2}$ while $\ell_{\text {rot }_{0}}(b)>0$ for $b \in\left(b_{1}, b_{2}\right)$. The set of $(a, b)$ in the resonance tongue with $b \in\left(b_{1}, b_{2}\right)$ is known as an "instability pocket". See [BS00, BPS03, PS06] and sections 4.1 and 4.3 for the analysis in the ODE periodic and quasi-periodic cases in the perturbative domain.

2.3. Numerical methods. We have computed the Lyapunov exponent $\beta(a, b)$ and the rotation number $\rho(a, b)$ (or some equivalent magnitude, see the remarks in Section 2.2) in different cases for more than hundred millions of couples $(a, b)$. Our main method has been the use of iterations of (2.1). Typically the computations are done in double precision arithmetic. The values of $\beta(a, b)$ and $\rho(a, b)$ are essential for all the items of the study.

Assume we start with an initial vector $v_{0}=\left(x_{0}, y_{0}\right)^{T}$ and $\theta_{0} \in \mathbb{T}^{d}$ so that $v_{k}$ are computed recurrently using (2.2). To prevent from overflow one has to scale $v_{k}$ from time to time. The main algorithm is as follows

- Choose an initial vector $v_{0} \in \mathbb{R}^{2}$ with $\left\|v_{0}\right\|_{2}=1$. Set $L=R=0$, select natural numbers $N_{1}, N_{2}, N_{3}, N_{4}$ and tolerances $\epsilon_{L}, \epsilon_{R}$ and start the iterations.

- After every iteration check if the $y$ component of $v$ has changed sign. If it does add 1 to $R$. After $N_{1}$ iterations compute the current norm $\|v\|_{2}$, normalise the current $v$ vector by dividing by $\|v\|_{2}$ and add $\log \left(\|v\|_{2}\right)$ to $L$, known as the "Lyapunov sum".

- After every $N_{1} \times N_{2}$ iterations store the current values of $L, R$. Then, when the number of iterates is a multiple of $N_{1} \times N_{2} \times N_{3}$ do a linear fit of the stored values of $L$ and $R$ using the last $50 \%, 30 \%$ and $10 \%$ of the data. Let the slopes obtained in this way be denoted as $s_{L}^{50}, s_{L}^{30}, s_{L}^{10}, s_{R}^{50}, s_{R}^{30}, s_{R}^{10}$, respectively. Then the computation is stopped if both

$$
\begin{aligned}
& m_{L}:=\max \left\{\left|s_{L}^{50}-s_{L}^{30}\right|,\left|s_{L}^{30}-s_{L}^{10}\right|\right\}<\epsilon_{L}, \\
& m_{R}:=\max \left\{\left|s_{R}^{50}-s_{R}^{30}\right|,\left|s_{R}^{30}-s_{R}^{10}\right|\right\}<\epsilon_{R} .
\end{aligned}
$$

Otherwise it continues up to a maximum of $N_{\max }=N_{1} N_{2} N_{3} N_{4}$ iterates. When the computation is stopped the values $s_{L}^{50}$ and $s_{R}^{50}$ are used as estimates of $\beta(a, b)$ and $\rho(a, b)$, respectively. The values of $m_{L}, m_{R}$ and $k /\left(N_{1} N_{2} N_{3}\right)$, where $k$ is the current number of iterates, are also stored.

Typical values for $v_{0}, N_{1}, N_{2}, N_{3}, N_{4}$ are $(1,0)^{T}, 100,10,1000,1000$ and for $\epsilon_{L}, \epsilon_{R}$ are $10^{-7}$ in the case of one frequency and $10^{-6}$ in case of two or more frequencies. See Section 4.2 for comments on alternative methods and different kinds of checks.

Using finite digits representation it is impossible to use the exact selected values for the components of $\omega$. Furthermore, to take the values of $n \omega \bmod 2 \pi$ would also 
require $\pi$ to be exactly represented. Both things give a small systematic error for a large number of iterates. Furthermore to speed up the computations the values of trigonometric polynomials in $\theta$ have been obtained by the recurrences of the $\cos (n \varphi)$ and $\sin (n \varphi)$ functions. Despite the recurrences are stable, this is affected by propagation of round off errors. All these errors are not so relevant taking into account the continuous dependence of the desired indicators as functions of $(a, b)$.

See [LSSW03, Sim09] and references therein for other algorithms that have also been tested.

Concerning the computation of the resonance tongues a bisection method has been used. If for given values of $(a, b)$ the computation of $\rho(a, b)$ has been done as described above with a tolerance $\epsilon_{R}$, the end points of the interval for given $b$ and $\rho_{0}$ are obtained by bisection by looking for zeros of $\rho-\left(\rho_{0} \pm m \epsilon_{R}\right)$, where $\rho_{0}$ is the value associated to the desired resonance. The value of $m$ is taken to ensure that the errors in the computation of $\rho$ do not give spurious estimates. In these computations we have used typically $\epsilon_{R}=10^{-8}$ and $m$ between 2 and 5 . The bisection is stopped when the $a$-interval which contains the zero is also less than $\epsilon_{R}$. Then $\rho$ is evaluated at values of $a$ in that interval with step size $\epsilon_{R} / 10$, as an additional check. Finally the computation is repeated changing $b$ with step size 0.01 , or smaller in selected domains.

From these computations not only the boundaries of the tongues are obtained, but also the amplitudes $\ell_{\text {rot }_{0}}(b)$, which allow to detect instability pockets and collapsed gaps.

\section{Results}

In our numerical study we decided to choose some representative models of discrete Schrödinger operators with one and several frequencies. For a single frequency the most studied model is, by far, the Almost Mathieu model, which is given by

$$
V(\theta)=\cos \theta
$$

and $\omega=2 \pi \gamma, \gamma$ being an irrational number that has been taken equal to the golden mean $(\sqrt{5}-1) / 2$ for most of the effective computations. This is a very particular model whose spectrum is symmetric with respect to $a=0$. In general it is immediate to check the

Proposition 3.1. Assume there exist an unimodular matrix $M$ and a constant vector $\varphi \in \mathbb{T}^{d}$ such that

$$
-V(\theta)=V(M \theta+\varphi)
$$

for all $\theta \in \mathbb{T}^{d}$. Then the spectrum is symmetric with respect to $a=0$.

As a less simple model we consider also the following modified potential

$$
V(\theta)=\cos \theta+\frac{1}{m}(\cos 2 \theta+\cos 3 \theta+\cos 4 \theta)
$$

which for $m \in(0,11)$ has several maxima and minima, in contrast with the Almost Mathieu model. Following the terminology used in [Sin87] we will refer to 3.2 as a non-Morse potential. For $m>11$ the potential (3.2) has only one maximum and one minimum, both non-degenerate. For two frequencies, we chose the model:

$$
V\left(\theta_{1}, \theta_{2}\right)=\cos \theta_{1}+\cos \theta_{2}, \quad \gamma=((\sqrt{5}-1) / 2, \sqrt{3}-1),
$$


which is the simplest generalisation of the Almost Mathieu model for two frequencies. For three frequencies we used the straightforward generalisation

$$
V\left(\theta_{1}, \theta_{2}, \theta_{3}\right)=\cos \theta_{1}+\cos \theta_{2}+\cos \theta_{3}, \quad \gamma=(\sqrt{2}-1, \sqrt{3}-1, \sqrt{5}-2) .
$$

Other $\gamma$ sets have been tested without detecting essential changes.

3.1. Rotation Numbers and Lyapunov exponents. The results for the Almost Mathieu case are illustrated in Figure 1. This case has to be considered as a check of the procedure. Due to the symmetry of (3.1), it is enough to consider $a \geq 0$ (see Proposition 3.1). For this computation and the ones displayed in Figures 2 to 5 the values of $N_{1}, N_{2}, N_{3}$ and $N_{4}$, see Section 2.3, are 100,10, 1000 and 1000, respectively. Data for the number of pixels, tolerances and about the output can be found in Table 1, which also contains data for the above mentioned figures.

In the green domains in Figure 1 (grey in the printed version) there is evidence that the operator is non-reducible. To check this property one looks for places where the Lyapunov exponent is positive and the rotation number is not constant.

Last two lines in Figure 1 show the evolution of the Lyapunov exponent and rot as a function of $a$ for $b=1,2$ and 4 . As is well known, for $b=2$ the spectrum has zero measure, the Lyapunov exponent is positive except in a set of zero measure and the rotation number is constant almost everywhere. On the other hand the values $b=1$ and $b=4$ are "dual" and their plots, letting aside changes of scale and a translation, are identical. An additional check will be presented in Section 3.2.

\begin{tabular}{rrrrrrrrrr}
\hline case & kpixels & $e_{L, R}$ & AI & MI & cases & $m_{L}>e_{L}$ & $m_{L} \max$ & $m_{R}>e_{R}$ & $m_{R} \max$ \\
\hline 3.1 & $4.0 \times 4.0$ & $1 . \mathrm{E}-7$ & 11.49 & 108 & 0 & 0 & $e_{L}$ & 0 & $e_{R}$ \\
3.2 & $6.0 \times 4.8$ & $1 . \mathrm{E}-7$ & 18.59 & 147 & 0 & 0 & $e_{L}$ & 0 & $e_{R}$ \\
3.3 & $8.0 \times 4.0$ & $1 . \mathrm{E}-6$ & 46.88 & 1000 & 9 & 9 & $0.9 \mathrm{E}-5$ & 0 & $e_{R}$ \\
3.4 & $5.3 \times 4.0$ & $1 . \mathrm{E}-6$ & 136.06 & 1000 & 231402 & 229859 & $3.5 \mathrm{E}-5$ & 72712 & $6.0 \mathrm{E}-6$ \\
\hline
\end{tabular}

TABLE 1. Data for the potentials (3.1) to (3.4). Details in the text.

In Table 1 the first column refers to the potential, the second one shows the kilopixels used in horizontal and vertical directions, $e_{L, R}$ denote the present tolerances, AI and MI stand for the average and maximal number of iterates (in millions). Then "cases" means for how many $(a, b)$ values one reaches $N_{\max }$ iterates and, after this, in how many cases one can not satisfy $m_{L} \leq \epsilon_{L}$ and the maximal $m_{L}$ found, and similar results for $m_{R}$. Anyway, in the (3.4) case, most computations exceeding $e_{L}$ have $m_{L}<10^{-5}$ and most computations exceeding $e_{R}$ have $m_{R}<2 \times 10^{-6}$.

As second example we present in Figure 2 the case (3.2) for $m=2$ and the golden mean. There is no symmetry and we have considered both negative and positive values of $a$. This example shows some remarkable features when compared to the Almost Mathieu Case.

- The Almost Mathieu model does not show any collapsed gap, as proved in [CEY90, AJ08] for Diophantine $\gamma$, whereas the case of (3.2) does show several collapsed gaps. There are two types of these collapsed gaps. When the Lyapunov exponent is zero, gaps may collapse for a single value of $b$ producing instability pockets as explained in Section 4.1. When the Lyapunov exponent is positive a different phenomenon appears, which is emphasized in the second and third rows of Figure 6. Some resonance tongues may collapse for an interval of $b$ values and then may reopen for a larger value 

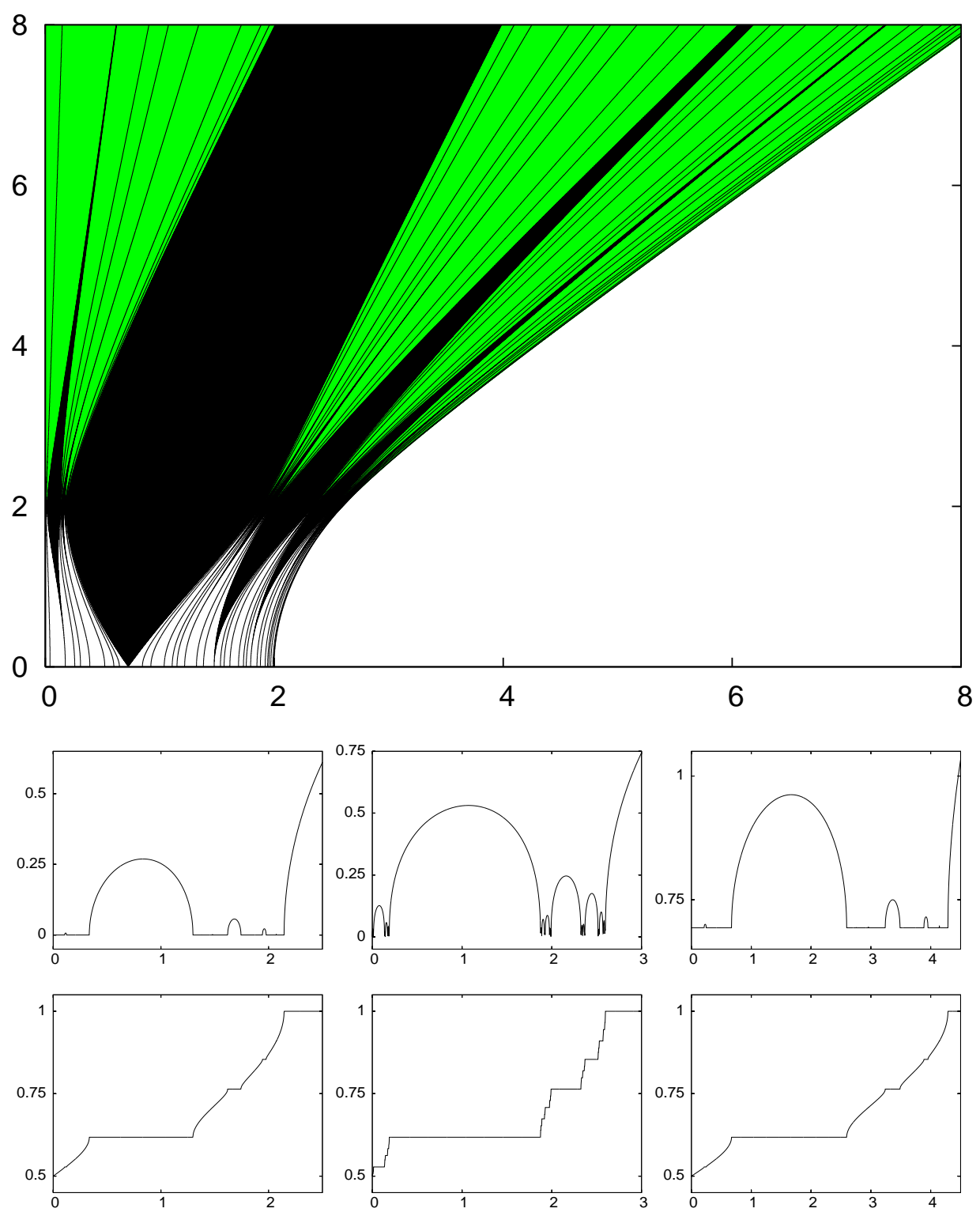

Figure 1. Top: Resonance tongues for the Almost Mathieu Model (3.1) and $\gamma_{1}=\frac{\sqrt{5}-1}{2}$. Last two bottom lines show Lyapunov exponent (upper line) and rot (lower line) for $b=1,2,4$ (from left to right) as a function of $a$. The green domains (grey in the printed version) correspond to values of $(a, b)$ for which one has evidence of lack of reducibility. To prevent from too many data in the plot, the order of the maximal resonance retained is 34 . This figure and subsequent ones allow for magnification in the electronic version. See text for details on the computations. 
of $b$. In all cases the collapse is sharp, in the sense that gap length tends linearly to zero at the tip of the tongue. In both cases, there are sufficiently many open spectral gaps to infer that the spectrum is a Cantor set.

- Concerning the regularity of tongue boundaries, as noted in Section 4.1, tongue boundaries are given by analytic functions for small values of $b$ and, thus, with zero Lyapunov exponent. When the Lyapunov exponent is positive, tongue boundaries are analytic for the Almost Mathieu case (this is a consequence of Aubry duality) whereas they seem to have non-smooth tips in the case of the potential (3.2). This seems to be related to the non-Morse character of the potential.

- In both cases, the Lyapunov exponent and the rotation number seem to be Hölder continuous but not differentiable at the endpoints of gaps.

- An interesting feature of (3.2) is the existence of ranges of $a$, say $\left(a_{1}, a_{2}\right)$, for which rot behaves almost linearly with a small slope but in which, definitely, rot is non constant, which end at places where a Hölder- $1 / 2$ behaviour is apparent at the left of $a_{1}$ and at the right of $a_{2}$.

Last two examples correspond to the potentials (3.3) and (3.4) with 2 and 3 frequencies, respectively, and the first results are shown in Figures 3 and 4 . Compared to the case with a single frequency, these examples have the following features:

- For $b$ large enough all gaps are collapsed and the spectrum consists of a single spectral band with positive Lyapunov exponent. At the point of collapse of a resonance tongue, the gap length tends to zero linearly. This non-smooth character also shows up at some places when the tongue is still open and the Lyapunov exponent is positive, the tongue boundaries being also non-smooth.

- When the Lyapunov exponent is positive, tongues which collapse do not seem to reopen again, in contrast with some tongues for the model (3.2). The behaviour with zero Lyapunov exponent is very different and there tongue boundaries are smooth and pockets may appear, see Section 4.1.

- When the Lyapunov exponent is positive, both the rotation number and the Lyapunov exponent seem to have one-sided derivatives (without the root behaviour at the endpoint of gaps observed in one frequency) and, whenever there is an spectral band in the spectrum with positive Lyapunov exponent, they seem to be smooth there.

Additional checks have been done for larger values of $b$, outside the ranges shown in the figures. To have "equivalent" behaviour (see also Section 3.2) we consider as parameter $\hat{b}=b \times|V|_{\infty}$. The values of $\hat{b}$ go from moderate, say $\hat{b}=20$, to as large as $\hat{b}=100$. The goal has been to detect the spectral gaps with a step $10^{-6}$ in $a$, step which has been decreased to $10^{-9}$ around the places where rot crosses a resonance. No relevant differences have been found between (3.1) and (3.2). For $\hat{b}=20$ very few gaps exceed the amplitude 0.1 a few more exceed 0.001 and most of the other gaps are below amplitude $10^{-6}$. On the other hand no trace of gaps has been found for (3.3) and (3.4) for $\hat{b} \geq 8$.

Figure 5 shows magnifications of Figures 2 and 3, to illustrate the existence of several instability pockets for (3.2) and the existence of almost tangential "contacts" between the different resonances (see Section 3.2 for more information). These contacts seem to define a separation between reducible and non-reducible domains 
outside the resonance tongues. On the top plot one can see some "holes" without almost contacts. But this is only due to the reduced number of resonances shown in the plot. The holes seem to disappear if resonances up to order 200 are retained, but then the figure will look as "fully black".
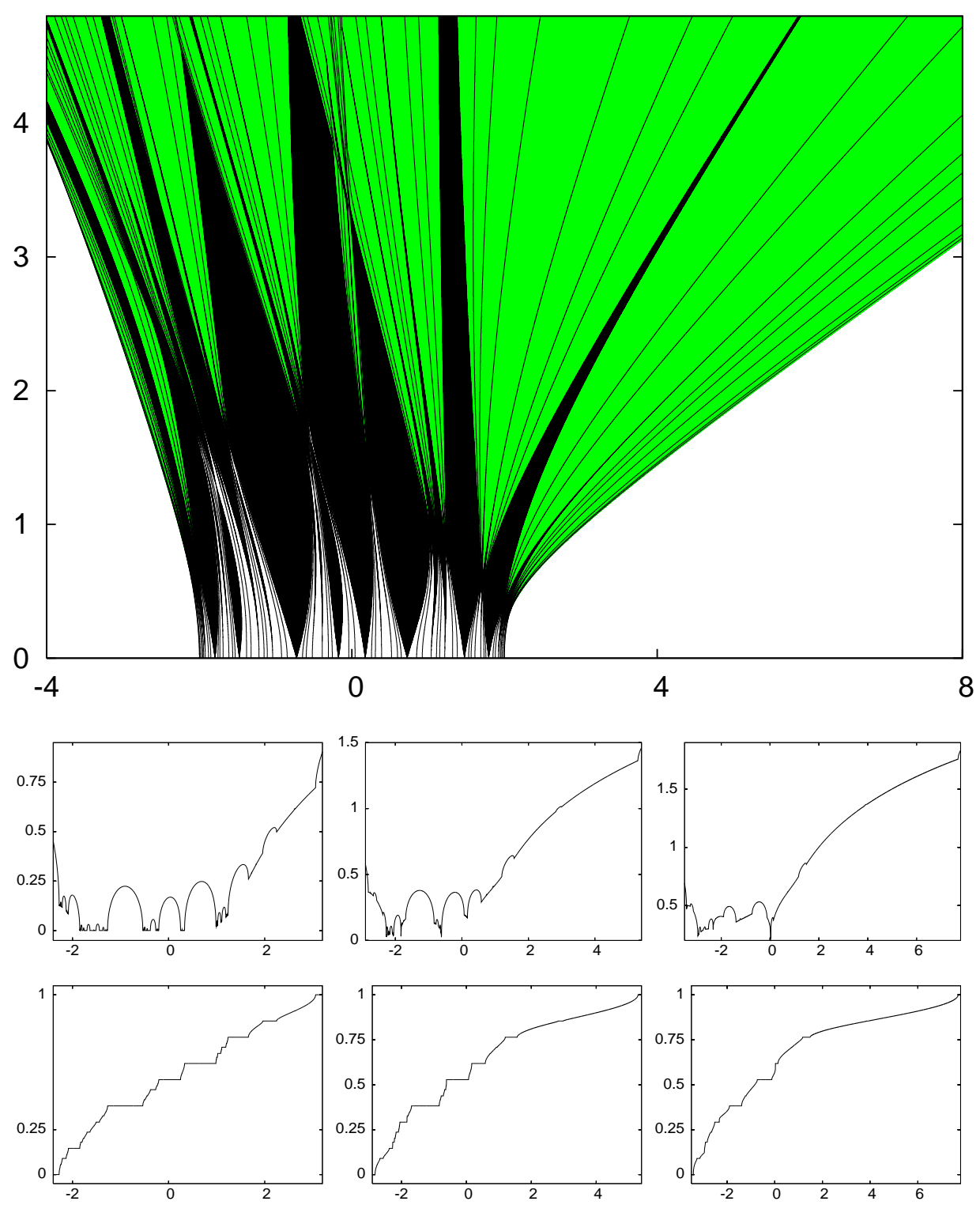

Figure 2. Top: Similar to Figure 1 for the one-frequency model (3.2) $\gamma_{1}=\frac{\sqrt{5}-1}{2}$ and $m=2$. Lyapunov exponent and rot displayed for $b=1,2,3$ (from left to right). Also only resonances up to order 34 have been retained. 
In Figure 6 we display the amplitudes $\ell_{\text {rot }_{0}}(b)$, see Section 2.2, for several resonances and potentials. This small sample illustrates the behaviour of different tongues and gives support to the list of items given before concerning differences between (3.1) and (3.2), from one side, and between the case of one frequency and more frequencies from the other.
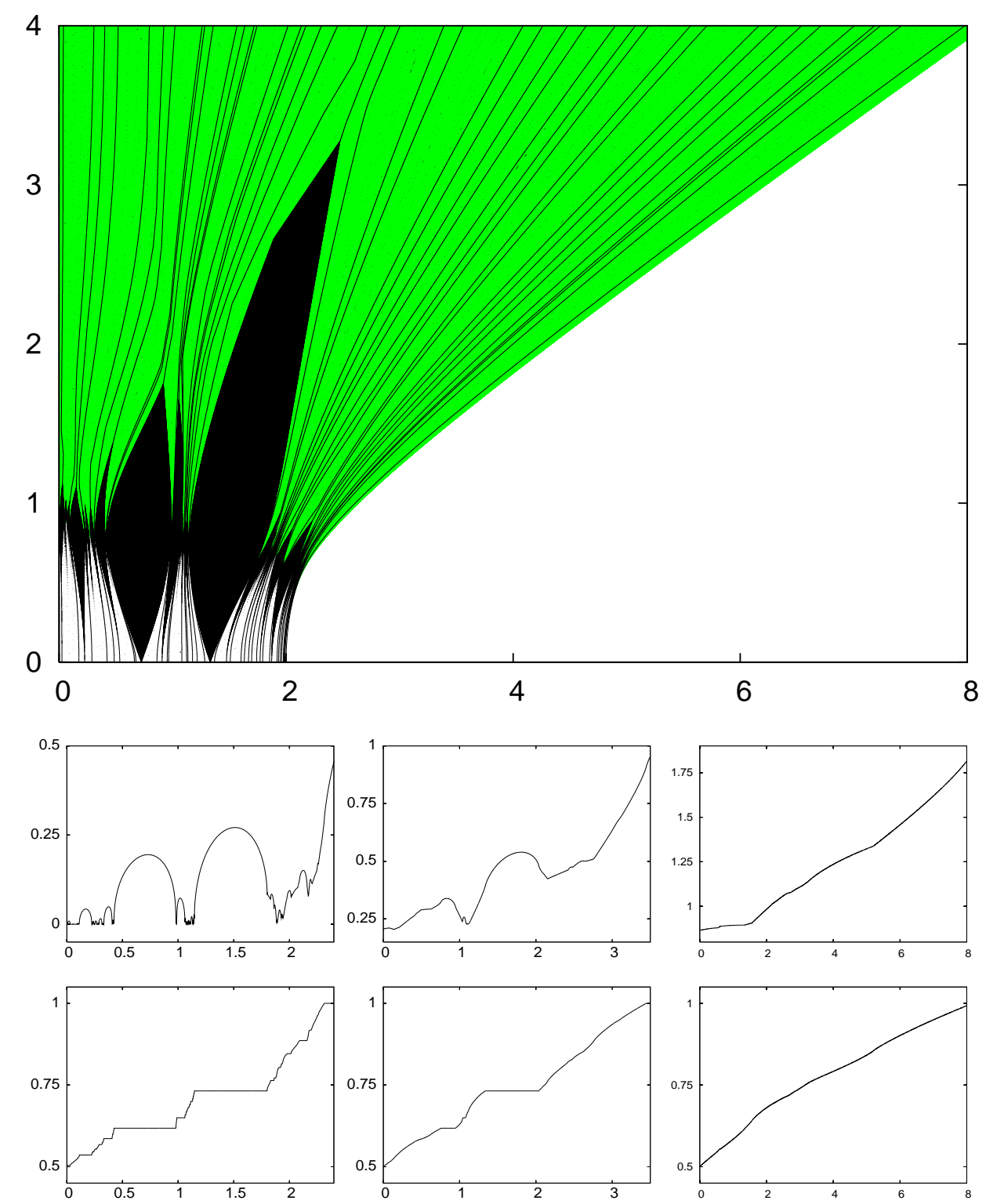

FIgURE 3. Top: Similar to Figure 1 for the two-frequency model (3.3) and $\gamma_{1}=(\sqrt{5}-1) / 2$ and $\gamma_{2}=\sqrt{3}-1$. Lyapunov exponent and rot displayed (from left to right) for $b=0.75,1.5,4$. All the resonances up to order 6 are shown and some selected resonances, with relatively large resonant zone, up to order 10. 

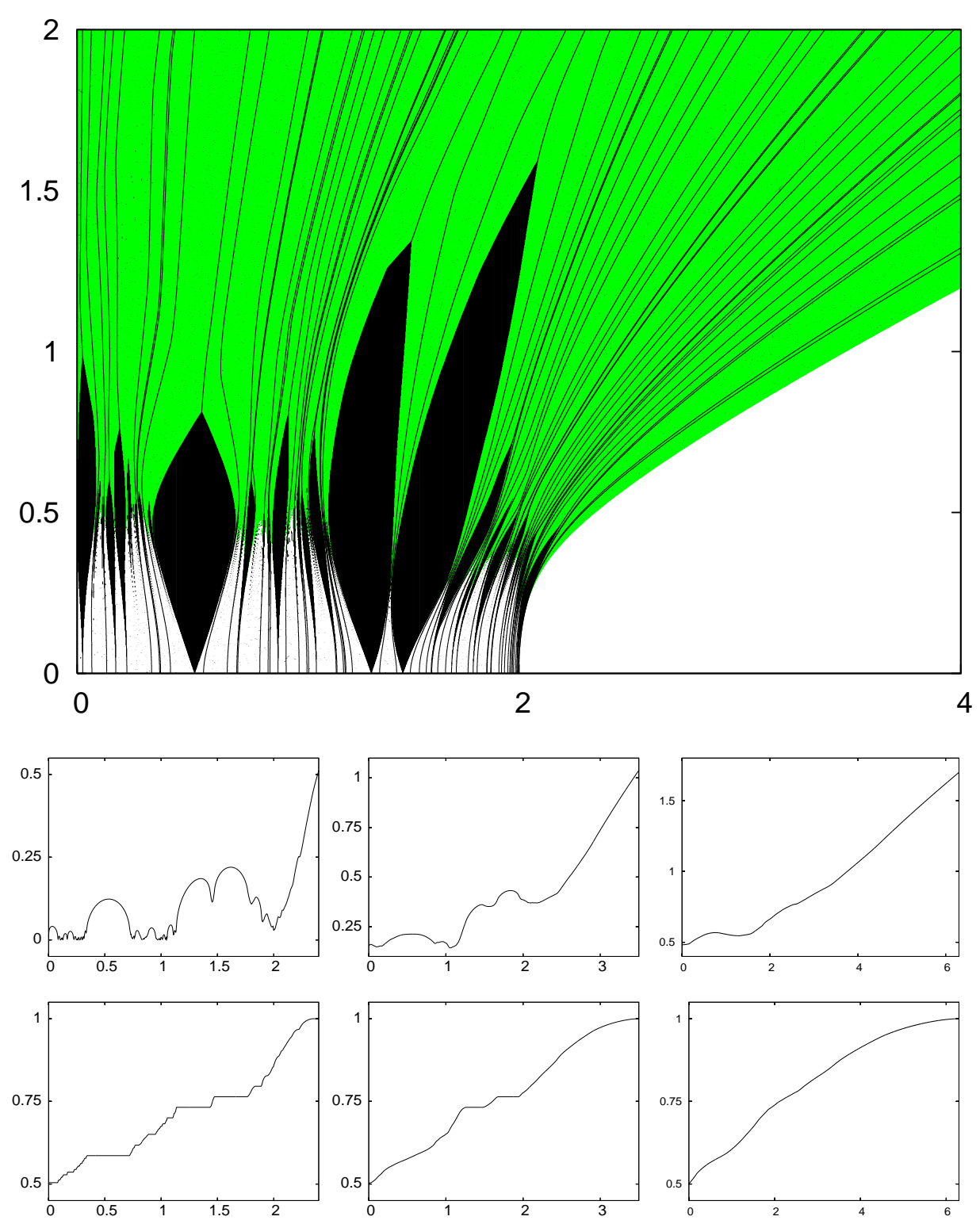

Figure 4. Top: Similar to Figure 1 for the three-frequency model (3.4) and $\gamma_{1}=\sqrt{2}-1, \gamma_{2}=\sqrt{3}-1, \gamma_{3}=\sqrt{5}-2$. Lyapunov exponent and rot displayed (from left to right) for $b=0.5,1,2$. All the resonances up to order 4 are shown and some selected resonances up to order 10 . Note that the computations have been done up to $a=8$ but only $a \in[0,4]$ is shown here for better detail. This has to be taken into account in Figure 7. 

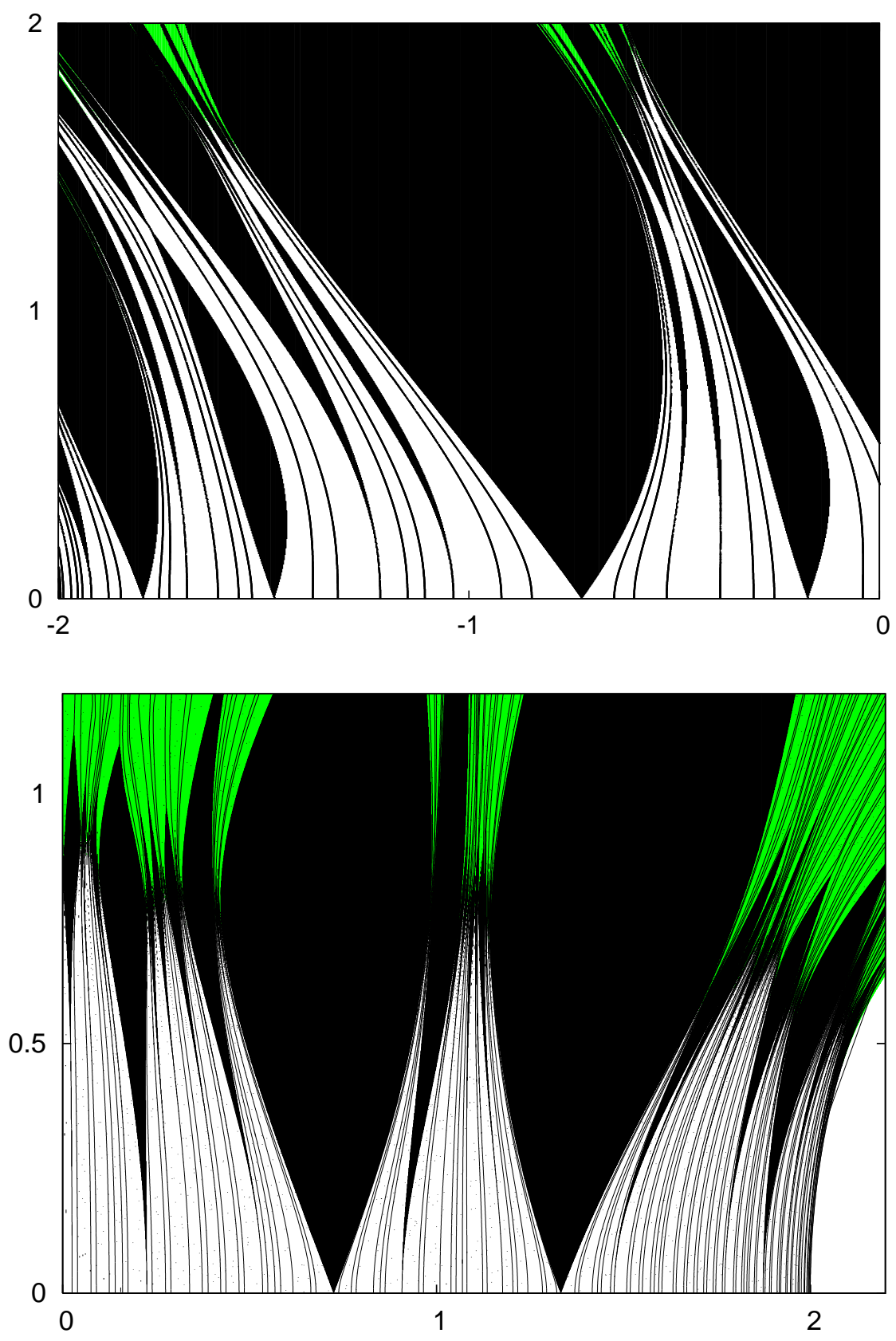

Figure 5. Magnifications of Figure 2 (top) and Figure 3 (bottom) showing details of tongues, pockets and resonances. On the top plot all the resonances up to order 34 which fall in the domain are retained. On the bottom one all the resonances to order 10 and some selected ones to order 20 are shown. 

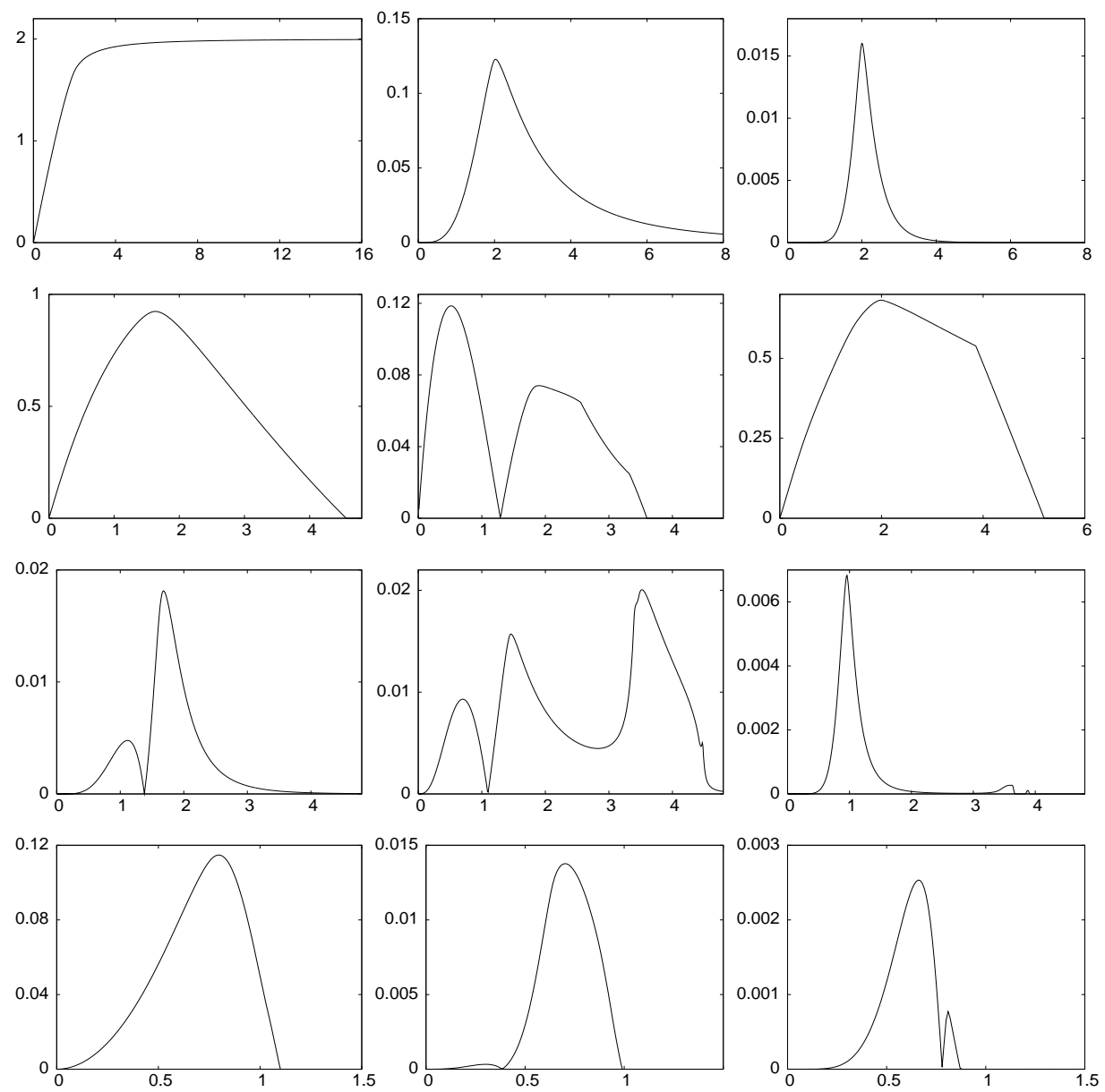

Figure 6. Amplitudes of a selected set of resonances for potentials (3.1), (3.2) and (3.3). The corresponding data are given in Table 2 below. They illustrate the different behaviour of non-collapsed and collapsed gaps, as well are the lack of smoothness of the tongue boundaries. The case (3.4) is quite similar to (3.3).

\begin{tabular}{r|r|r|r||r|r|r|r||r|r|r|r}
\hline case & $\mathbf{k}$ & $k_{0}$ & rot & case & $\mathbf{k}$ & $k_{0}$ & rot & case & $\mathbf{k}$ & $k_{0}$ & rot \\
\hline 3.1 & 1 & 0 & 0.618034 & 3.1 & -4 & 3 & 0.527864 & 3.1 & -10 & 7 & 0.819660 \\
3.2 & -1 & 1 & 0.381966 & 3.2 & 2 & -1 & 0.236068 & 3.2 & -4 & 3 & 0.527864 \\
3.2 & -14 & 9 & 0.347524 & 3.2 & 9 & -5 & 0.562306 & 3.2 & -20 & 13 & 0.639320 \\
3.3 & $0,-2$ & 2 & 0.535898 & 3.3 & $0,-3$ & 3 & 0.803848 & 3.3 & $-2,4$ & -1 & 0.692135 \\
\hline
\end{tabular}

TABle 2. Data for Figure 6. The table is ordered in the same way as the plots in the figure. For every plot we identify the case and then the coefficients $\mathbf{k}, k_{0}$ in rot $=\langle\mathbf{k}, \gamma\rangle+k_{0}$, according to the Gap Labelling, and the approximate value of rot. 

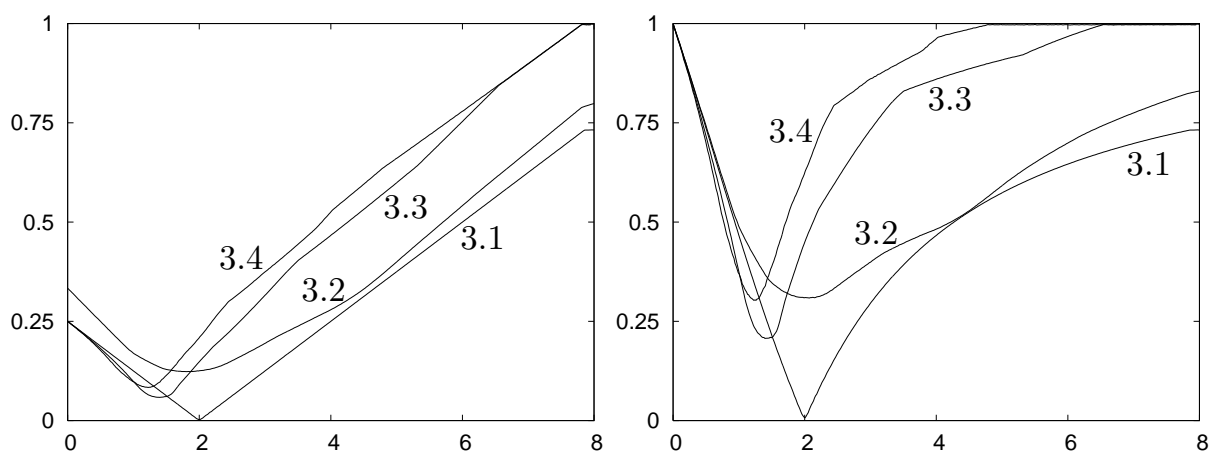

FiguRE 7 . The plots display the measure of the spectrum as a function of $b \times|V|_{\infty}$ for the cases studied, (3.1), (3.2), (3.3) and (3.4). On the left we plot an "absolute" value, that is, the estimated measure of the spectrum in the $a$ interval explored divided by the amplitude of this interval. This means that we divided by 8 in the symmetric cases and by 12 in the non-symmetric case (3.2). In particular, when $b=0$ the scaled values are $1 / 4$ and $1 / 3$, respectively. On the right we plot a "relative" value, that is the measure of the spectrum divided by the amplitude of the range of $a$ between the left and right uniformly hyperbolic zones. All resonances up to order 100 have been used for (3.1) and (3.2), to order 20 for (3.3) and to order 10 for (3.4).

3.2. Measure of the spectrum. A natural question to ask is what is the Lebesgue measure of the spectrum of a quasi-periodic Schrödinger operator (1.1) as a function of $b$. When $b=0$, the spectrum is the set $[-2,2]$ and the measure is thus 4 . In the Almost Mathieu case, there is a general formula for the measure of the spectrum

$$
\left|\sigma_{b}\right|_{\text {Leb }}=|4-2| b||
$$

as shown in a series of works, see [Jit07] for references. A very particular feature of the Almost Mathieu case is that when $b= \pm 2$ the spectrum has zero measure. This does not happen in general, as seen in Figure 7 for the models under consideration and can be understood looking at the structure of resonance tongues. Indeed, for each boundary of a resonance tongue, there seems to be a height $b$ for which nearby resonance tongues have almost tangential contacts. This height of $b$ is precisely the value of $b$ for which the Lyapunov exponent, restricted to the tongue boundary, "takes off" from $\beta=0$ to positive values. In the $(a, b)$-parameter plane, the set of such critical points (defined only in the spectrum) seems to lie along a curve, which in the Almost Mathieu case is $b=2$ but, in general is not horizontal, even far from horizontal, see related results [Avi09, HP]. Thus, although resonance tongues cluster when the Lyapunov exponent becomes positive, this happens in general at different values of $b$ and the measure of the spectrum is in general positive. The existence of that curve was already detected in the numerical experiments in [BS98] for the continuous case.

Note that, along this critical curve, the measure of the spectrum (i.e. values of $(a, b)$ not in the interior of resonance tongues) is zero. A related result [AK06] states that in one frequency (inside a full measure class of Diophantine numbers), 
$V$ real analytic and for a fixed value of $b$, then for Lebesgue almost every value of $a$, the cocycle $\left(A_{a, b}, \omega\right)$ is either reducible to a rotation (and thus in the spectrum) or has positive Lyapunov exponent. Since cocycles along the critical line cannot be reducible to a rotation and have zero Lyapunov exponent, along any such horizontal line (e.g. $b=2$ in the Almost Mathieu case), the intersection with the spectrum would have zero Lebesgue measure.

Figure 7 also shows that the measure of the spectrum decreases linearly around $b=0$ (gaps appear when $b \neq 0$ ) and also grows linearly with $b$ as $b \rightarrow \infty$. Both limit cases can be studied by refined perturbative techniques of reducible systems [Eli92, HA09, AJ08] and diagonal operators [Eli97, Bje05].

\section{Discussion}

In this paper we have numerically explored the structure of the spectrum of onedimensional Schrödinger operators with a quasi-periodic potential having one or more frequencies, with an eye on the differences that this multifrequency character makes on the structure. As an additional example we have also explored the case of a single frequency with a potential that has several minima and maxima. We have numerically observed that, for small values of $b$ our models have basically all gaps open and zero Lyapunov exponent in the spectrum. When $b$ grows the Lyapunov exponent becomes positive at a certain height and gaps start decreasing in size. When $b$ grows further and there are many frequencies, gaps collapse in a non-smooth way and stay collapsed for larger values of $b$, whereas in the case with a single frequency, there seems to be a dense set of open spectral gaps (coexisting with collapsed gaps in some cases). In this section we want to discuss some of these different properties.

4.1. Perturbative gap opening. As we see from Figures 1-5, the behaviour for small values of $b$ is similar and, although some differences, can be understood using a common framework, which, incidentally, is also similar to the periodic case.

For $b=0$, the rotation number can be directly computed (since the cocycle is in constant coefficients) and it is seen that resonance tongues emanate from the points in the $b=0$ axis of the form

$$
a_{\mathbf{k}}= \pm 2 \cos (\langle\mathbf{k}, \omega\rangle \pi), \quad \mathbf{k} \in \mathbb{Z}^{d} .
$$

The cases $\mathbf{k}=\mathbf{0}$ correspond to the maximum $(a=2)$ and minimum $(a=-2)$ of the spectrum in the trivial $b=0$ case. The cases $\mathbf{k} \neq \mathbf{0}$ correspond to the tips at $b=0$ of tongue-shaped regions in the $(a, b)$-plane which, for every value of $b$ are given by an interval $\left[a_{\mathbf{k}}^{-}(b), a_{\mathbf{k}}^{+}(b)\right]$ which may collapse.

Using perturbative techniques and reducibility results it is possible to understand the structure of resonance tongues for small values of $|b|$ and a fixed analytic potential $V$ as it was done in [BPS03] for the continuous analog (described briefly in 4.3). Let us give the main ideas in this discrete context.

Let $a_{\mathbf{k}}= \pm 2 \cos (\langle\mathbf{k}, \omega\rangle \pi)$ be one of the resonant values of $a$ for $b=0$ and $\mathbf{k} \neq 0$. Then

$$
A_{a_{\mathbf{k}}, 0}=\left(\begin{array}{cr}
a_{\mathbf{k}} & -1 \\
1 & 0
\end{array}\right)
$$

and, since all solutions are quasi-periodic with frequency $\omega$, after a quasi-periodic change of variables, $Z_{\mathbf{k}}$, the system reduces to the identity when $b=0$ and $a=a_{\mathbf{k}}$. 
Introducing new local coordinates $\delta=a-a_{\mathbf{k}}$ then the cocycle $\left(A_{a, b}, \omega\right)$ reduces to one of the form

$$
B(\theta, \delta, b)=I d+Z_{\mathbf{k}}^{-1}(\theta+\omega)\left(\begin{array}{cc}
\delta+b V(\theta) & 0 \\
0 & 0
\end{array}\right) Z_{\mathbf{k}}(\theta)
$$

which is a perturbation of the identity. After $r$ steps of averaging, this can be analytically reduced to a cocycle $\left(B^{r}, \omega\right)$ of the form

$$
B^{r}(\theta, \delta, b)=M^{r}(\delta, b)+P^{r+1}(\theta, \delta, b)
$$

where the remainder, $P^{r+1}$, which contains the $\theta$-dependence, is of order $r+1$ in $(\delta, b)$ and $M^{r}$ is a matrix independent of $\theta$ with $M^{r}(0,0)=I d$. As in the continuous case, the normal form up to order $r$ can be used to derive the Taylor expansions $a=a_{\mathbf{k}}^{ \pm}(b)$ of tongue boundaries up to order $r$ around $(a, b) \equiv\left(a_{\mathbf{k}}, 0\right)$. In particular, the order of tangency for every tongue can be computed performing a finite number of averaging steps and, for example, tongues with index $\mathbf{k}$ and $V_{\mathbf{k}} \neq 0$, the $\mathbf{k}$-th Fourier coefficient of $V$, are transversal at the origin. For potentials like the Almost Mathieu (3.1) or containing its two and three-dimensional analogues, like (3.3) and (3.4), the order of tangency at $b=0$ of the $\mathbf{k}$-th tongue is, at least $|\mathbf{k}|$, and sometimes greater than this value, see Appendix B in [BPS03].

For small values of $|b|$, Eliasson's reducibility theory applies [Eli92, HA09] and tongue boundaries can be seen to be smooth functions of $b$, whose Taylor expansions around $b=0$ can be effectively computed. This allows to create examples which display instability pockets (i.e. tongue boundary crossings) at small values of $b$. Besides, for a generic real analytic $V$, all gaps are open for small values of $|b|$ [Eli92, BPS03, Pui06]. For the analytic character of the tongue boundaries for $b$ small one can adapt the methods of [PS06] to the discrete case.

4.2. Accuracy of Numerical Computations. As the study carried out in this paper is based on numerical explorations, we have tried to do different kinds of extensive checks. They can be mainly classified as follows.

1) As described in Section 2.3 most of the computations have been done up to a maximal number of iterates $N_{\max }=10^{9}$ and internal coherence between estimates of $\beta$ and $\rho$ equal to $\epsilon_{L}$ and $\epsilon_{R}$. Our primordial interest is on the accuracy of $\rho$. Hence we have carried out checks with different values of $\epsilon_{R}$ and $N_{\max }$ while $\epsilon_{L}$ has been taken larger than $\epsilon_{R}$ (say, $\epsilon_{L}=100 \epsilon_{R}$ ) in order that most of the computations stop before $N_{\max }$ iterates.

Almost in all cases the observed "error" in $\rho$, taking as correct the one obtained with many more iterates (up to $10^{12}$ ) is below the requested $\epsilon_{R}$, and in all cases is less than $2 \epsilon_{R}$. The results are worse for $\beta$. Despite in a large part of the cases the observed error (in the above sense) is below $\epsilon_{L}$, in a few cases it reaches $10 \epsilon_{L}$. As it is natural the largest errors are found for the largest values of $b$ that we have explored.

2) As mentioned, the value of the phase $\phi$ is irrelevant for the computation of $\beta$ and $\rho$. This fact can be used to produce different estimates obtained by using different initial phases. We proceed as in Section 2.3 using the slopes $s_{L}^{50}$ and $s_{R}^{50}$ as estimators and going always to a number of iterates equal to $N_{\max }$. Then one can compute the average and standard deviation of a sample obtained with equispaced $\phi \in[0,2 \pi)$.

The method has been implemented and tested for several ranges of $a$ and different $b$ values in the studied examples. Using $N_{\max }=10^{7}$ and samples 
of size 100 the average $\left\langle s_{R}^{50}\right\rangle$ differs from the value obtained as described in Section 2.3 using $N_{\max }=10^{9}$ by less than $\epsilon_{R}$. Furthermore the standard deviation $\sigma_{s_{R}^{50}}$ is of the order of $2 \epsilon_{R}$.

3) One can proceed by a direct approximate computation of the spectrum as follows. Consider a symmetric tridiagonal matrix $G_{\phi}$ whose elements are

$$
g_{n, n}=b V(\omega n+\phi), \quad g_{n, n+1}=g_{n+1, n}=1 .
$$

The spectrum of the Schrödinger operator is the spectrum of the "infinite matrix" $G_{\phi}$ when $n \in \mathbb{Z}$. One can "truncate" $G_{\phi}$ between $n_{1}$ and $n_{2}$ (for instance $\left.n_{1}=0, n_{2}=10^{6}\right)$ to obtain an approximation $G_{\phi,\left[n_{1}, n_{2}\right]}$ of the operator and approximations of both the rotation number and the Lyapunov exponent can be computed from the eigenvalues, obtained using a QR method adapted to band matrices, from this truncation. In fact, each of the four elements of the transfer matrix

$$
M_{a, b}^{n}(\theta)=A_{a, b}(\theta+(n-1) \omega) \cdots A_{a, b}(\theta),
$$

which is a polynomial in $a$ and $b$ can be expressed as a determinant of $G_{\phi,\left[n_{1}, n_{2}\right]}-a I$ for suitable values of $\phi, n_{1}$ and $n_{2}$. In particular, the first column is formed by the determinants of $G_{\theta,[0, n]}-a I$ and $G_{\theta,[0, n-1]}-a I$.

Carrying out explicit computations for the different examples and a selected sample of values of $b$ one checks that, with the resolution provided by this direct method, the gaps coincide with the ones determined using the rotation number.

4) When collapsed gaps are detected, say for $a=a_{c}$ for a given $b$, a range of the form $\left(a_{c}-\delta, a_{c}+\delta\right)$ around $a_{c}$ has been scanned with a small step size in $a$, small $\epsilon_{R}$ and large $N_{\text {max }}$. Typical values for $\delta$ range from $10^{-6}$ to $10^{-9}$. It has been checked that $\rho$ behaves in a strict monotonous way around $a_{c}$ without numerical traces of gap.

As additional checks some one frequency cases have been tested, like (3.2) for some $m>11$ or $W(\theta)=W(\theta)-\bar{W}$, where $W(\theta)=\exp (\cos (\theta)), \bar{W}=$ $\frac{1}{2 \pi} \int_{0}^{2 \pi} W(\theta) d \theta$. The potential $V(\theta)=\cos (\theta)+\frac{1}{2} \sin (2 \theta)+\frac{1}{4} \sin (4 \theta)$ has also been checked. For last two cases the symmetry applies and it is enough to explore $a \geq 0$. As value of $\gamma_{1}$ either the golden mean or other quadratic irrationals have been tested. In these Morse cases the behaviour is similar to the one found in Almost Mathieu. On the other hand, model (3.2) for $m<11$, going up to $m=1$ looks like the $m=1 / 2$ case in what concerns collapsed gaps and subsistence of very narrow resonant zones for $b$ large.

We want to add some final comments on bounds of the errors in the computation of Lyapunov exponent and rotation number. As clearly mentioned in [DV05] there are three main sources of error: a) the ones due to the algorithm in the computation, as analysed in [DV05] when using QR methods; b) the ones due to round-off (or in the continuous case due to the truncation errors of the integration method) and its propagation; c) the ones due to the "convergence to the limit". Last one is, in most cases, the most important and difficult to estimate, particularly in the nonuniformly hyperbolic zones, where no a priori estimates are known. Several ways to "extrapolate to the limit" are presented in [BS98, LSSW03, Sim09] and references therein, and a slightly different version has been used in present paper and in [PS10]. Finally, let us mention that inside resonance tongues, in the uniformly hyperbolic 
domain, it is possible to use computer assisted proofs to provide rigourous bounds for the Lyapunov exponent $[\mathrm{FH}]$.

An indication of the convergence to the limit can be obtained as follows. Let $L_{n}$ be the Lyapunov sum after $n$ iterates, see Section 2.3, and $f_{N}(n)$ a linear fit to the values of $L_{n}$ up to $n=N$. Then let $d(N)=\max \left\{\left|L_{n}-f_{N}(n)\right|, n \leq N\right\}$. Note that, as $f_{N}$ changes with $N$ the function $d(N)$ is not necessarily monotonically increasing. While in the reducible case $d(N)$ turns out to be bounded, and hence the errors in the computation of $\beta(a, b)$ are at most $\mathcal{O}\left(N^{-1}\right)$, in the non-reducible case values up to $d(N)=\mathcal{O}\left(N^{1 / 4}\right)$ have been observed. See [PS10] for details and illustrations. Hence, with the algorithm used in present paper we can be confident on errors in $\beta(a, b)$ below $\mathcal{O}\left(10^{-6}\right)$ in the reducible domain and at most below $\mathcal{O}\left(10^{-5}\right)$ in the non.reducible one. The results are better, typically by a factor of 10 , for the rotation number. When the computations for an $(a, b)$ region are completed, a "quick" way to guess the non-reducible domain can be to look at the number of iterates needed to stop the computations. If with the tolerances used (see Section 2.3 ) it exceeds, say, a value like $10^{8}$ for some values of $(a, b)$, one can be confident that the system is non-reducible there.

4.3. Extension to the continuous case. An important companion model to discrete Schrödinger operators are their continuous analogues, whose eigenvalue equations are second order differential equations of the form

$$
x^{\prime \prime}(t)+b Q(\omega t+\phi) x(t)=a x(t)
$$

where $Q: \mathbb{T}^{d} \rightarrow \mathbb{R}$ is a quasi-periodic function with frequency vector $\omega$. Note that, in contrast with the discrete case the periodic case occurs with $d=1$. The perturbative situation has been studied in [Eli92, JS92, BPS03, PS06] and the situation is similar to the discrete case. It is clear that the study of (4.1) can be reduced to a discrete case if we use the "map time one of the periods" (say, the time- $2 \pi / \omega_{1}$-map) which depends on the other frequencies. However, while in (2.1) the role of $(a, b)$ is linear, in the continuous case it can be highly non-linear. Another strong difference with (2.1) is that, while in the discrete case for a given value of $b$, no matter how large it is, the spectrum stops at a sufficiently large value of $a$, in (4.1) for large $a$ most of the values belong to the spectrum.

The situation for larger values of $b$ has been studied by [FSW90] for a model and [Bje06] for more general analytic potentials, yielding positivity of the Lyapunov exponents for sufficiently large $b$ and $a$ in the bottom of the spectrum. The existence of gaps in this setting was considered in a particular example by [FJP02]. For an analytic and numerical study in the case of two frequencies we refer to [BS98] which contains a detailed account of efficient numerical methods in the continuous case and the relation with other problems. An extension of this work to the case of three frequencies can be found in [PS10]. The results for this continuous case are similar to the ones found in the present study for the discrete version. Furthermore, details on the maximal deviation $d(N)$ of the current Lyapunov and rotation sums, $L$ and $R$, and the values given by a linear fit, are shown as a function of the number of iterates $N$. Some hints on a relation between $d(N)$ and the simultaneous Diophantine properties of the frequencies are also given.

Finally we want to stress that, beyond the spectral problems in Schrödinger operators, equations of the type (4.1) appear in the study of the stability of invariant tori, as normal variational equations along a quasi-periodic solution. They are a 
natural generalisation of the classical Hill equations which appear in the periodic case. Furthermore, more general equations arise in higher dimensional normal variational equations. Most of the problems in this case, away from perturbative ones, are open.

4.4. A summary of detected phenomena. We summarize here some of the phenomena observed in this numerical study.

(1) The separation between the reducible and non-reducible domains in $(a, b)$ has a complicated structure. A wild line seems to separate both domains. That line was simply $b=2$ in the Almost Mathieu case. Of course, the line can be drawn in different ways inside the uniformly hyperbolic zones.

(2) In contrast with the case of one frequency, the collapse of resonances is produced in a sharp way when there are two or more frequencies. Before reaching the collapse, the width of resonance tongues behaves almost linearly. Some of the collapsed tongues can reopen and have a definitive collapse for larger values of $b$.

(3) In the nonuniformly hyperbolic zones, the boundaries of the resonant tongues seem to be nondifferentiable, with lateral derivatives, at some points.

(4) In the case of several frequencies and even for moderate values of $b$, the spectrum seems to have no gaps inside.

All these phenomena should be checked on a variety of examples and analysed theoretically.

\section{REFERENCES}

[Avi09] A. Avila. Global theory of one-frequency Schrödinger operators I: stratified analyticity of the Lyapunov exponent and the boundary of nonuniform hyperbolicity. Arxiv preprint arXiv:0905.3902, 2009.

[ABD09] A. Avila, J. Bochi, and D. Damanik. Cantor spectrum for Schrödinger operators with potentials arising from generalized skew-shifts. Duke Math. J., 146(2):253-280, 2009.

[AJ05] A. Avila and S. Jitomirskaya. The Ten Martini Problem. Ann. of Math., 170(1):303343, 2009.

[AJ08] A. Avila and S. Jitomirskaya. Almost localization and almost reducibility. J. Eur. Math. Soc., 12(1):93-131, 2010.

[AK06] A. Avila and R. Krikorian. Reducibility or non-uniform hyperbolicity for quasiperiodic Schrödinger cocycles. Ann. of Math., 164(3):249-294, 2006.

[Bje05] K. Bjerklöv. Positive Lyapunov exponent and minimality for a class of one-dimensional quasi-periodic Schrödinger equations. Ergodic Theory Dynam. Systems, 25(4):10151045, 2005.

[Bje06] K. Bjerklöv. Positive Lyapunov exponents for continuous quasiperiodic Schrödinger equations. J. Math. Phys., 47:022702, 2006.

[BMS76] N. Bogoljubov, J. A. Mitropoliskii, and A. M. Samoŭlenko. Methods of accelerated convergence in nonlinear mechanics. Hindustan Publishing Corp., Delhi, 1976.

[BPS03] H. W. Broer, J. Puig, and C. Simó. Resonance tongues and instability pockets in the quasi-periodic Hill-Schrödinger equation. Comm. Math. Phys, 241(2-3):467-503, 2003.

[BS98] H. W. Broer and C. Simó. Hill's equation with quasi-periodic forcing: resonance tongues, instability pockets and global phenomena. Bol. Soc. Brasil. Mat. (N.S.), 29(2):253-293, 1998.

[BS00] H. W. Broer and C. Simó. Resonance tongues in Hill's equations: a geometric approach. J. Differential Equations, 166(2):290-327, 2000.

[CEY90] M. D. Choi, G. A. Elliott, and N. Yui. Gauss polynomials and the rotation algebra. Invent. Math., 99(2):225-246, 1990. 
[CS89] V. A. Chulaevsky and Ya. G. Sinal̆. Anderson localization for the 1-D discrete Schrödinger operator with two-frequency potential. Comm. Math. Phys., 125(1):91$112,1989$.

[CF08] N. D. Cong and R. Fabbri. On the spectrum of the one-dimensional Schrödinger operator. Discrete Contin. Dyn. Syst., Ser. B, 9(3-4):541-554, 2008.

[DJ87] C. De Concini and R.A. Johnson. The algebraic-geometric AKNS potentials. Ergodic Theory Dynam. Systems, 7(1):1-24, 1987.

[DS83] F. Delyon and B. Souillard. The rotation number for finite difference operators and its properties. Comm. Math. Phys., 89(3):415-426, 1983.

[DV05] L. Dieci and E. S. Van Vleck. On the error in computing Lyapunov exponents by QR Methods. Numer. Math., 101:619-642, 2005.

[Eli92] L. H. Eliasson. Floquet solutions for the one-dimensional quasi-periodic Schrödinger equation. Comm. Math. Phys., 146:447-482, 1992.

[Eli97] L. H. Eliasson. Discrete one-dimensional quasi-periodic Schrödinger operators with pure point spectrum. Acta Math., 179(2):153-196, 1997.

[FJP02] R. Fabbri, R.A. Johnson, and R. Pavani. On the nature of the spectrum of the quasiperiodic Schrödinger operator. Nonlinear Anal. Real World Appl., 3(1):37-59, 2002.

[FH] J.L. Figueras and À. Haro. Computer Assisted Proofs of existence of fiberwise hyperbolic invariant tori in skew products over rotations. In preparation, 2010.

[FSW90] J. Fröhlich, T. Spencer, and P. Wittwer. Localization for a class of one-dimensional quasi-periodic Schrödinger operators. Comm. Math. Phys., 132(1):5-25, 1990.

[GS08] M. Goldstein and W. Schlag. Fine properties of the integrated density of states and a quantitative separation property of the Dirichlet eigenvalues. Geom. Funct. Anal., 18(3):755-869, 2008.

[HA09] S. Hadj Amor. Hölder Continuity of the Rotation Number for Quasi-Periodic Co-Cycles in. Comm. Math. Phys., 287(2):565-588, 2009.

[HP] À. Haro and J. Puig. Aubry duality for quasi-periodic potentials: Lyapunov exponents and Cantor spectrum. In preparation.

[Her83] M.R. Herman. Une méthode pour minorer les exposants de Lyapunov et quelques exemples montrant le caractère local d'un théorème d'Arnold et de Moser sur le tore de dimension 2. Comment. Math. Helvetici, 58(3), 1983.

[Jit07] S. Jitomirskaya. Ergodic Schrödinger operators (on one foot). Gesztesy, Fritz et al. (ed.), Spectral theory and mathematical physics. A festschrift in honor of Barry Simon's 60th birthday. Ergodic Schrödinger operators, singular spectrum, orthogonal polynomials, and inverse spectral theory. Based on the SimonFest conference, Pasadena, CA, USA, March 27-31, 2006. Providence, RI: American Mathematical Society (AMS). Proceedings of Symposia in Pure Mathematics 76, Pt. 2, 613-647 (2007)., 2007.

[Joh91] R.A. Johnson. Cantor spectrum for the quasi-periodic Schrödinger equation. J. Diff. Eq., 91:88-110, 1991.

[JM82] R.A. Johnson and J. Moser. The rotation number for almost periodic potentials. Commun. Math. Phys., 84:403-438, 1982.

[JS92] À. Jorba and C. Simó. On the reducibility of linear differential equations with quasiperiodic coefficients. J. Differential Equations, 98(1):111-124, 1992.

[Kin68] J.F.C. Kingman. The ergodic theory of subadditive stochastic processes. J. Roy. Statist. Soc. Ser. B, 30:499-510, 1968.

[LSSW03] F. Ledrappier, M. Shub, C. Simó, and A. Wilkinson. Random versus deterministic exponents in a rich family of diffeomorphisms. J. Stat Phys., 113:85-149, 2003.

[MW79] W. Magnus and S. Winkler. Hill's equation. Dover Publications Inc., New York, 1979. Corrected reprint of the 1966 edition.

[MP84] J. Moser and J. Pöschel. An extension of a result by Dinaburg and Sinai on quasiperiodic potentials. Comment. Math. Helvetici, 59:39-85, 1984.

[Pui04] J. Puig. Cantor spectrum for the Almost Mathieu operator. Comm. Math. Phys, 244(2):297-309, 2004.

[Pui06] J. Puig. A nonperturbative Eliasson's reducibility theorem. Nonlinearity, 19(2):355376, 2006.

[PS06] J. Puig and C. Simó. Analytic families of reducible linear quasi-periodic differential equations. Ergodic Theory Dynam. Systems, 26(2):481-524, 2006. 
[PS10] J. Puig and C. Simó. Resonance tongues in the Quasi-Periodic Hill-Schrödinger Equation with three frequencies. Regul. Chaot. Dyn., to appear.

[SS78] R. J. Sacker and G. R. Sell. A spectral theory for linear differential systems. J. Diff. Eq., 27:320-358, 1978.

[SNS09] J. Sánchez, M. Net, and C. Simó. Computation of invariant tori by Newton-Krylov methods in large-scale dissipative systems. Physica D, 239:123-133, 2010.

[Sim09] C. Simó. Some properties of the global behaviour of conservative low dimensional systems. In Foundations of Computational Mathematics: Hong Kong 2008, volume 363 of London Math. Soc. Lecture Note Ser., pages 163-189. Cambridge Univ. Press, Cambridge, 2009.

[Sin87] Ya. G. Sinai. Anderson localization for one-dimensional difference Schrödinger operator with quasiperiodic potential. J. Statist. Phys., 46(5-6):861-909, 1987.

Departament de Matemàtica Aplicada i. Universitat Politècnica de Catalunya. DiaGONAL, 647. 08028 BARCELONA (SPAin).

E-mail address: joaquim.puig@upc.edu

Departament de Matemàtica Aplicada i Anàlisi. Universitat de Barcelona. Gran Via de les Corts Catalanes, 585. 08007 Barcelona (Spain).

E-mail address: carles@maia.ub.es 